\title{
Ärztliche Beiträge an die berufliche und soziale Wiedereingliederung von Patienten
}

\author{
C. A. Ludwig
}

Die Wiedereingliederung von Patientinnen und Patienten in ihr soziales und berufliches Umfeld ist ohne ärztliche Unterstützung undenkbar. Um der zunehmenden Komplexität der damit verbundenen Aufgaben gerecht zu werden, kommen vermehrt neuartige Instrumente der Behandlungsführung, wie etwa das Fallmanagement, zum Einsatz. Um mit den anderen Betreuungsinstanzen optimal zusammenarbeiten zu können, müssen Ärztinnen und Ärzte über versicherungsmedizinisches Basiswissen verfügen und Supportdienste zu nutzen wissen. Swiss Insurance Medicine, die schweizerische Interessengemeinschaft für Versicherungsmedizin, hat eine Initiative mit dem Schwerpunkt «Arbeitsfähigkeitsbeurteilung» lanciert. Sie will dazu beitragen, dass die Ärzte über die nötige Fachkenntnis verfügen, damit sie auch weiterhin kompetent bei der Reintegration von Patienten mitwirken können.

Trotz der Bemühungen der am Versorgungsprozess Beteiligten steigt die Zahl der Personen, welche den Weg zurück zur Arbeit nur verzögert, nur teilweise oder überhaupt nicht mehr finden. Dies mit schwerwiegenden Folgen nicht nur für die betroffenen Patienten und deren Familien, sondern auch für die Volkswirtschaft [1]. Dass die Wiedereingliederung sich tendenziell immer schwieriger gestaltet, hat wesentlich mit den Veränderungen im beruflichen und sozialen Umfeld zu tun. Oft verhindert auch die Fragmentierung der Betreuungsprozesse, wie sie mit der fortschreitenden Spezialisierung in der Rehabilitation einhergeht, eine gut abgestimmte Patientenbetreuung.

\section{Einfluss der weichen Faktoren}

Nicht nur die medizinische Behandlung, sondern zahlreiche weitere Faktoren entscheiden darüber, ob eine Reintegration gelingt. So können Schadenfälle zu medizinisch nicht plausibler Invalidität eskalieren, wenn bei den Patienten Unsicherheit über den Erhalt ihres Arbeitsplatzes und das berufliche Fortkommen herrscht oder wenn diese ihre Stelle bereits verloren haben. Auch vom Patienten als bedrohlich wahrgenommene Arbeitsplatzbedingungen können eine Wiedereingliederung erschweren. Die Bemühungen scheitern gehäuft bei alleinerziehenden Müttern, Migrantinnen und Migranten, bei Patienten mit psychischen Problemen oder solchen mit geringem Bildungsstand. Ebenso können zeitliche Verzögerungen im Rehabilitationsprozess dazu führen, dass eine Wiederaufnahme der Arbeit misslingt. Alle Beteiligten müssen neben den biologischen auch die psychosozialen und beruflichen Faktoren kennen, damit wirksame Massnahmen gefunden und erfolgreich durchgeführt werden können. Der Aufwand, den es für derartig umfassende Abklärungen und für koordinative Aufgaben zu leisten gilt, übersteigt allerdings die Ressourcen eines behandelnden Arztes. Kostenträger und andere Einrichtungen haben deshalb begonnen, in solchen Fällen unterstützend einzugreifen.

\section{Integrierte Betreuung dank Fallmanagement}

So betreibt die Schweizerische Versicherungsanstalt (Suva) ein neuartiges Betreuungssystem, dank welchem der Anteil reintegrierter Patientinnen und Patienten erhöht werden soll. Kernstück bildet das Programm «New Case Management». Dieses sieht - je nach Komplexität der bei den einzelnen Patienten vorliegenden Problemeeine differenzierte Fallbearbeitung vor. Denjenigen Patienten, bei denen psychische oder soziale Probleme erkannt werden, nimmt sich ein Case-Manager aus der zuständigen Versicherungsagentur an. Dieser führt in enger Zusammenarbeit mit dem lokalen Kreisarzt, den behandelnden Ärzten und den weiteren Beteiligten eine Standortbestimmung durch. Aufgrund der daraus gewonnenen Erkenntnisse über das individuelle Rehabilitationspotential werden Interventionen geplant und aufeinander abgestimmt. Der Fallmanager berät seine Patienten im Zusammenhang mit der Beanspruchung von Versorgungsdienstleistungen wie auch in beruflichen, versicherungstechnischen und finanziellen Angelegenheiten. Das «New Case Management» hat eine Neuorientierung der Suva-Versicherungsmedizin erforderlich gemacht [2]. Die Kreisärzte des Agenturärztlichen Dienstes der Suva engagieren sich stärker als früher in der Rehabilitationsberatung [3]. Um psychische Störungen rechtzeitig erfassen und diesbezügliche therapeutische Massnahmen einleiten zu 
können, baut die Suva einen Versicherungspsychiatrischen Dienst auf. In mehreren Agenturen stehen bereits Konsiliarpsychiater zur Verfügung [4]. Das Kompetenzzentrum Versicherungsmedizin der Suva in Luzern unterstützt die Betreuungspersonen und unterhält einen Auskunftsdienst [5]. Weitere wichtige Elemente in der Versorgungskette sind die Rehabilitationskliniken und Einrichtungen, welche die funktionelle Leistungsfähigkeit und die Berufseignung evaluieren. Auch Berufsberatungs- und Arbeitsvermittlungsstellen sowie Sozialdienste müssen regelmässig in Anspruch genommen werden. Und schliesslich tragen die Absenzenmanagementprogramme in den Firmen dazu bei, dass die Arbeitnehmenden entsprechend ihren Fähigkeiten schrittweise in ihren Tätigkeitsbereich zurückgeführt werden können. Mittels einer Mitte 2004 angelaufenen Kampagne möchte die Suva das Bewusstsein in der Bevölkerung stärken, dass eine gescheiterte oder verzögerte Reintegration mit hohen Kosten verbunden ist. Die Arbeitgeber will man motivieren, die nötigen betrieblichen Rahmenbedingungen zu schaffen, die den Mitarbeitenden eine Rückkehr in die Berufstätigkeit ermöglichen [1].

\section{Herausforderungen für die behandelnden Ärzte}

Ärzte sind innerhalb des Wiedereingliederungsmanagements nicht nur als Therapeuten tätig, sondern übernehmen auch versicherungsmedizinische und gutachterliche Aufgaben. Dann etwa, wenn sie sich zur Arbeitsfähigkeit einer Patientin oder eines Patienten zu äussern haben oder wenn es gilt, bleibende gesundheitliche Beeinträchtigungen sowie die daraus resultierenden Behinderungen oder die zumutbaren Arbeitsleistungen zu beurteilen. Solche Expertentätigkeit setzt voraus, dass man das Umfeld und die jeweiligen Belastungen am Arbeitsplatz des Patienten kennt. Dies kann schwierig sein, sind doch Arbeitnehmende oft in Bereichen tätig, die mit dem ursprünglichen Beruf kaum mehr etwas gemeinsam haben. Bei Teilzeitbeschäftigten oder Arbeitslosen kann sich die Festlegung des Arbeitsfähigkeitsgrades als besonders kompliziert erweisen. Hinzu kommt, dass Ärzte gelegentlich Druckversuchen von Patienten, welche ein Gefälligkeitszeugnis einfordern, ausgesetzt sind. Sachbearbeitende in den Versicherungsgesellschaften haben den Eindruck, dass manch ein Arzt seinen versicherungsmedizinischen Aufgaben nicht gewachsen ist. Bestehende Supportangebote, wie Fallmanagement- oder
Absenzenmanagementprogramme, Auskunftsdienste oder die Inspektorendienste der Versicherer scheinen zuwenig bekannt $\mathrm{zu}$ sein und bleiben oft ungenutzt.

\section{Initiative «Arbeitsfähigkeitsbeurteilung»}

Swiss Insurance Medicine (SIM), die schweizerische Interessengemeinschaft für Versicherungsmedizin, will zur Verbesserung der ärztlichen Kompetenz in diesem Aufgabenkreis beitragen. Die Fachgesellschaft hat zu diesem Zweck bereits die einschlägigen Bildungsangebote erfasst und in einem Katalog zusammengestellt [6]. Eine SIM-Arbeitsgruppe hat im Frühjahr 2004 einen Workshop organisiert, an welchem über dreissig Fachleute, nämlich Ärztinnen und Ärzte aus Spital und Praxis sowie Vertreter der Sozialversicherungen und der Privatassekuranz, teilnahmen. Bei dieser Gelegenheit wurden die Bedürfnisse der Ärzte und der Kostenträger im Zusammenhang mit dem Wiedereingliederungsmanagement eruiert. Vordringlicher Handlungsbedarf zeigte sich bei den Formularen, mittels welchen Arbeitsunfähigkeit attestiert wird. Diese sollten nach Ansicht der Workshopteilnehmenden verbessert und standardisiert werden. Es sollten zusätzliche Informationen darin aufgenommen werden, welche die Abschätzung von Arbeitsfähigkeit erleichtern. Es wurde vorgeschlagen, in Zusammenarbeit mit den Sozialpartnern ein Instrumentarium zur Früherfassung von Arbeitsunfähigkeit zu schaffen. Man war sich darin einig, dass es nötig ist, den Ärzten regelmässig versicherungsmedizinisches Wissen zu vermitteln und entsprechende Bildungsangebote bereitzustellen. Die SIM nimmt sich prioritär der Aufgabe an, ein einheitliches Arbeitsfähigkeitsattest zu schaffen. Ein Fortbildungsmodul speziell zur Arbeitsfähigkeitsbeurteilung befindet sich in Entwicklung; es soll interessierten Ärztegesellschaften, Kliniken und Kongressorganisatoren angeboten werden.

\section{Kooperation im Wiedereingliederungs- management}

Verständlicherweise ist es das vordringliche Anliegen von Medizinalpersonen, ihre Patienten vor allem in der körperlichen und psychischen Rehabilitation zu unterstützen. Der Betreuungserfolg nach einem Unfall oder nach einer Krankheit muss aber auch daran gemessen werden, ob die betroffenen Menschen ihren Platz im sozialen und beruflichen Umfeld wiederfinden. Ärzte 
können dann wesentlich zu einer umfassenden Wiederherstellung beitragen, wenn sie über die nötige versicherungsmedizinische Kompetenz verfügen und wenn ihnen die enge Zusammenarbeit mit allen anderen Akteuren gelingt. Die laufenden Initiativen sind geeignet, wichtige Voraussetzungen dafür zu schaffen und damit die Wiedereingliederungschancen vieler Patienten $\mathrm{zu}$ verbessern.
Literatur

1 Morger W. Kosten der Versicherungsleistungen. Medizinische Mitteilungen (Suva) 2004;75:5-18

2 Ludwig CA. Versicherungsmedizin im Wandel. Medizinische Mitteilungen (Suva) 2003;74:5-7.

3 Ermanni M. Der Agenturärztliche Dienst der Suva. Medizinische Mitteilungen (Suva) 2004;75:40-2.

4 Hoffmann-Richter U. Versicherungspsychiatrischer Dienst der Suva. Medizinische Mitteilungen (Suva) 2004:75:48-50.

5 Versicherungsmedizinischer Auskunftsdienst der Suva: Tel. 0414195239 (Mo-Fr: 8.00-17.00 Uhr).

6 Swiss Insurance Medicine (Hrsg.). Bildungskatalog Versicherungsmedizin 2004/2005. Winterthur: Eigenverlag; 2004 (Bezug über SIM-Geschäftsstelle, E-Mail: markus.bonelli@bonelli.ch).

\title{
Aus-, Weiter- und Fortbildung in Versicherungsmedizin und für die Festlegung der Arbeitsunfähigkeit
}

\author{
B. Soltermann
}

Bei der Festlegung der Arbeitsunfähigkeit nimmt die Ärzteschaft eine versicherungsmedizinische Vermittlerrolle zwischen den Patienten, den Versicherern und den Arbeitgebern ein. Obwohl diese Arbeitsunfähigkeiten die Versicherungen in der Schweiz pro Jahr rund 4 Milliarden Franken kosten, sind keine diesbezüglichen Lernziele in der Aus- und Weiterbildung der Ärzte formuliert. Insbesondere bei unklaren Arbeitsunfähigkeiten sollte möglichst rasch Klarheit geschaffen werden; dies könnte durch versicherungsmedizinische Arbeitsfähigkeitsabklärungsstellen AFAS geschehen. In einem zukünftigen versicherungsmedizinischen Netzwerk mit Universitäten und Fachhochschulen sollten Fähigkeitsausweise oder ein Master of Insurance Medicine erworben werden können.

\footnotetext{
a Unter Versicherten sind nicht nur Patientinnen und Patienten gemeint, sondern alle Prämien-
}

Korrespondenz:

Dr. med. Bruno Soltermann Schweizerischer

Versicherungsverband SVV Conrad F. Meyer-Strasse 14 CH-8022 Zürich

E-Mail: bruno.soltermann@svv.ch

\section{Versicherungsmedizin}

Die Versicherungsmedizin beschäftigt sich mit den Beziehungen zwischen den Versicherten ${ }^{\mathrm{a}}$, den Versicherern ${ }^{\mathrm{b}}$, den Leistungserbringern ${ }^{\mathrm{c}}$, den Arbeitgebern und allenfalls auch den Juristen $^{\text {d }}$. Es handelt sich hierbei um eine Interdisziplinarität, in welcher die Ärzteschaft, insbesondere versicherungsmedizinisch tätige Ärzte, eine Vermittlerrolle einnehmen müssen.

\section{Versicherungsmedizin und Attestierung der Arbeitsunfähigkeit}

Jeder Arzt, sei er nun Hausarzt, Spitalarzt, Versicherungsarzt, der ein Arbeitsunfähigkeitszeugnis ausstellt, nimmt eine versicherungsmedizinische Vermittlerrolle zwischen seinem Patienten, dem Versicherer sowie dem Arbeitgeber ein.

Um die volle Arbeitsunfähigkeit oder die Restarbeitsfähigkeit beurteilen zu können, muss der Arzt die Anforderungen an den Patienten an seinem Arbeitsplatz kennen. Der Arzt muss nicht Berufsberater sein, aber er muss durch eine gezielte Befragung herausfinden, welche Bewegungsabläufe nötig sind und welchen Belastungen der Körper ausgesetzt werden muss, um den Beruf ausüben zu können, oder welche geistigen Anforderungen und Konzentrationsintensitäten benötigt werden.

Wenn man bedenkt, dass in der Schweiz jährlich rund 4 Milliarden Franken an Taggeldern der Unfallversicherer und Lohnfortzahlungen bei Krankheit ausbezahlt werden [1], so ist es doch erstaunlich, dass im Medizinstudium der Schweiz kein Lernziel betreffend der Festlegung 
zahler in einer Solidargemeinschaft, welche mit ihren Prämien gesamthaft für die Schäden aufkommen müssen, so dass eine gerechte Entschädigung jenen zu gewährleisten ist, denen sie zusteht, da ansonsten die Prämien mehr und mehr steigen.

b Unter Versicherern versteht man Kranken-, Unfall-, Taggeld-, Lebens- und Haftpflichtversicherer wie auch die Invaliden- und die Militärversicherung.

c Ärzte, Physiotherapeuten, Labors, Spitäler.

d Patientenanwälte, Versicherungsjuristen, Richter. der Arbeitsunfähigkeit respektive der Arbeitsfähigkeit formuliert ist [2], und auch in den Weiterbildungsordnungen der Fachgesellschaften wird diesem Thema kaum Aufmerksamkeit geschenkt.

Seit 1998 werden unter der Schirmherrschaft der FMH Kurse für ärztliche Begutachtung im Bereich der Unfallversicherung durchgeführt, in welchen an Plenarvorträgen und Workshops die Festlegung der Arbeitsunfähigkeit resp. die Zumutbarkeit unterrichtet und geübt wird. Bis heute haben knapp 400 Ärztinnen und Ärzte (Spitalärzte aller Hierarchiestufen, frei praktizierende Ärzte sowie Versicherungsmediziner) an diesen Kursen teilgenommen. Der nächste Begutachtungskurs findet im Mai 2005 in Zürich statt, dannzumal unter der Mitwirkung der Swiss Insurance Medicine SIM.

\section{Vorschlag der Invalidenversicherung zur Reduzierung der Langzeitarbeits- unfähigkeit}

Angesichts der steigenden Ausgaben im Taggeldund Rentenbereich macht sich die Invalidenversicherung IV Gedanken, wie sie möglichst rasch die Versicherten erfassen kann. Vorgeschlagen wird, ein System zur Früherkennung und Begleitung (FEB) zu schaffen [3]. Die Aufgaben der FEB sollen durch spezielle Fachstellen wahrgenommen werden. Diese können von den betroffenen Versicherten selbst, vom Arbeitgeber, vom behandelnden Arzt oder auch von der Taggeldversicherung des Arbeitgebers bei längerer oder wiederkehrender Abwesenheit vom Arbeitsplatz eingeschaltet werden. Aufgabe der Fachstelle wäre in erster Linie, mit allen Beteiligten Kontakt aufzunehmen, gegebenenfalls beim regionalen ärztlichen Dienst der IV eine medizinische Abklärung zu veranlassen und bei den zuständigen Organen die Massnahmen in Gang zu setzen, die geeignet wären, den Betroffenen möglichst weitgehend den Verbleib im Erwerbsleben zu ermöglichen.

\section{Vorschlag der Swiss Insurance Medicine zur Reduzierung der Langzeitarbeits- unfähigkeit}

Die Swiss Insurance Medicine möchte noch einen Schritt weitergehen und ist mit einer Projektgruppe daran, versicherungsmedizinische Arbeitsfähigkeitsabklärungsstellen AFAS zu definieren. Das Ziel sollte sein, Versicherte mit attestierter, aber fraglicher Arbeitsunfähigkeit inner- halb von 6 Wochen einer solchen AFAS zuzuleiten, um den Reintegrationsprozess möglichst rasch und effizient zu gewährleisten. Eine effiziente versicherungsmedizinische AFAS erlaubte es, Leistungsansprüche gerecht und objektiv abzuklären. Sie ersparte den Versicherern, aber auch Versicherten und der Solidargemeinschaft aller Versicherten unnötige Kosten für Abklärungen, Therapien, Rehabilitation, Renten und andere geldwerte Leistungen, liesse andererseits den wirklich Berechtigten die ihnen zustehenden Leistungen unverzüglich zukommen. Eine gute Triage, in welchen Fällen eine Rehabilitation Sinn machen würde und in welchen nicht, diente der optimalen Ressourceneinteilung. Ziel müsste sein, nicht nur die Arbeits(un)fähigkeit festzulegen, sondern eine versicherungsmedizinische Beurteilung zu erstellen, welche die wahren Gründe der Arbeitsunfähigkeit fände und juristische, soziale und psychische Faktoren einbeziehen würde. In einer solchen AFAS bräuchte es versicherungsmedizinisch geschulte Ärzte verschiedenster Fachrichtungen, insbesondere psychosomatisch ausgerichtete Ärzte, daneben müssten aber auch Berufsberater und CaseManager involviert werden.

Hausärzte würden durch solche AFAS entlastet, denn die Bestimmung der Arbeits(un)fähigkeit insbesondere bei unklaren Verhältnissen belastet die Hausarzt-Patienten-Beziehung.

\section{Weiter- und Fortbildung in Versicherungsmedizin}

Die Weiter- und Fortbildung in Versicherungsmedizin muss Ärzte in sämtlichen Fachrichtungen in genügender Zahl erreichen, damit es nicht zu Staus in den AFAS kommt.

Dasselbe gilt natürlich auch für die vielen Gutachten, die mehr und mehr verlangt werden, sei dies nun wegen der Arbeits(un)fähigkeit, der Erwerbsfähigkeit, der medizinisch-theoretischen Invalidität, der Integritätsentschädigung, der Kausalitätsfragen, der Abgrenzungen Unfall/ Krankheit oder wegen Haftpflichtfällen.

Leider sind heutzutage die meisten Gutachter überlastet. Ein halbes Jahr bis ein ganzes Jahr Wartefrist für eine Begutachtung sind häufig. Qualitätsmängel der Gutachten sind nicht selten, da sie den Anforderungen nicht genügen [4].

Viele Assistenzärzte und Oberärzte in den Kliniken wie auch viele Ärzte in der Praxis sind nicht erpicht auf Gutachten. Die Gründe hierfür sind mannigfaltig; zum einen fehlendes versicherungsmedizinisches Wissen und kaum diesbezüglich fachliche Unterstützung, zum ande- 
ren müssen Gutachten oder medizinische Beurteilungen in der Freizeit, also abends oder am Wochenende, erstellt werden. Ein nicht zu unterschätzender Faktor besteht darin, dass der Arzt in der gutachterlichen Situation seinen Hauptauftrag, nämlich sich voll und ganz dem Wohl des Patienten zu widmen, ablegen und dafür die objektiven und subjektiven Aspekte klar voneinander trennen und die verschiedensten Gesichtspunkte unter einen Hut bringen muss; denn als beauftragter medizinischer Sachverständiger steht der Arzt im Brennpunkt unterschiedlichster Forderungen und Interessen, wie z.B. der wissenschaftlichen Lehre, der gesetzlichen Bestimmungen und Rechtsprechung, der legitimen Erwartungen des Auftraggebers, des Versicherten selber, allenfalls mit seinem Rechtsvertreter und seinem behandelnden Arzt, und nicht selten auch des Arbeitgebers.

Swiss Insurance Medicine will das versicherungsmedizinische Wissen weiterverbreiten. Ein erster Schritt wurde bereits durch die Schweizerische Gesellschaft der Vertrauensärzte SGV gemacht. Seit 2003 kann der Fähigkeitsausweis Vertrauensarzt erlangt werden, indem ein Weiterbildungskurs (4 Module) besucht werden muss [5]. Da dieser Fähigkeitsausweis jedoch das Hauptaugenmerk auf die Krankenversicherung legt, sind die Aspekte der übrigen Versicherungszweige (Unfall, Haftpflicht, Leben, IV, MV) für einen allfälligen Weiterbildungskurs Versicherungsmedizin verstärkt zu berücksichtigen.

\section{Versicherungsmedizinische Akademie in einem Netzwerk}

Swiss Insurance Medicine ist bestrebt, eine versicherungsmedizinische Akademie im Sinne eines Netzwerkes zu errichten, in welcher Versicherungsmedizin in klinischer und theoretischer Form gelehrt wird, daneben aber auch auf die gesundheitsökonomischen, versicherungsjuristischen und ethischen Aspekte eingegangen werden kann. Swiss Insurance Medicine ist daran, mit Interessenten von Universitäten und Fachhochschulen die Zusammenarbeit und Realisierung zu prüfen. Ein Abschluss mit einem Fähigkeitsausweis Versicherungsmedizin oder einem Mastertitel, wie dies übrigens auch in der EU vorgesehen ist, muss auch in der Schweiz erstrebenswert sein. Die Vision von gesamteuropäischen Standards in Versicherungsmedizin ist nicht mehr unerreichbar, wie dies die Schwerpunktnummer der Schweizerischen Ärztezeitung vom August 2004 über die Europäische Tabelle zur Bewertung der Beeinträchtigung der physischen und psychischen Integrität aufzeigt [6]. Die Europäische Union hat durch Versicherungsmediziner verschiedenster Länder diese Europäische Tabelle auf der Bezugsbasis zum täglichen Leben und der zwingenden Objektivierbarkeit im Konsens erstellt. Gleiche Funktionseinbussen werden gesamteuropäisch versicherungsmedizinisch gleich bewertet. Der Feinheitsgrad der Tabelle erfordert eine verbesserte und vertiefte Ausbildung der versicherungsmedizinischen Experten. Eine Übernahme der Europäischen Bewertungstabelle durch die Schweiz wäre aus medizinischer und versicherungsmedizinischer Sicht zu befürworten. Prof. Rudolf Kissling schreibt zu Recht, dass auch hier die Swiss Insurance Medicine das Heft in die Hand nehmen sollte.

\section{Literatur}

1 Bundesamt für Statistik. Jahre 1990 bis 2002.

2 Ackermann-Liebrich U, Egger M, Gutzwiller F, Paccaud F, Rougemont A, Weiss S, Westkämper R. Lernziele der Sozial- und Präventivmedizin (inkl. Arbeitsmedizin und Versicherungsmedizin) im Medizinstudium.

3 Mediendokumentation des Bundesamtes für Sozialversicherung zur 5. IV-Revision vom 28. April 2004. Weichenstellung des Bundesrates: Rentenzunahme abbremsen - Anreize zur Integration verbessern - Sparen.

4 Soltermann B. Aufbau eines medizinischen Gutachtens. Mitteilungen der Lebensversicherer an die Schweizer Ärzteschaft 2001;(27):16-20.

5 Zollikofer J. Fähigkeitsausweis Vertrauensarzt. Schweiz Ärztezeitung 2003;84(24):1267-9.

6 Kissling R. Europäische Tabelle zur Bewertung der Beeinträchtigung der physischen und psychischen Integrität der Confédération européenne d'experts en évaluation du dommage corporel CEREDOC. Schweiz Ärztezeitung 2004;85(35): 1841-3. 